\title{
Increased Brahma-related Gene 1 Expression Predicts Distant Metastasis and Shorter Survival in Patients with Invasive Ductal Carcinoma of the Breast
}

\author{
SUNG-IM DO ${ }^{1}$, GUN YOON ${ }^{2}$, HYUN-SOO KIM ${ }^{3}$, KYUNGEUN KIM ${ }^{1}$, HYUNJOO LEE ${ }^{1}$, \\ IN-GU DO ${ }^{1}$, DONG-HOON KIM ${ }^{1}$, SEOUNG WAN CHAE ${ }^{1}$ and JIN HEE SOHN ${ }^{1}$ \\ ${ }^{1}$ Department of Pathology, Kangbuk Samsung Hospital, \\ Sungkyunkwan University School of Medicine, Seoul, Republic of Korea; \\ ${ }^{2}$ Shinsegae Women's Hospital, Daegu, Republic of Korea; \\ ${ }^{3}$ Department of Pathology, Severance Hospital, Yonsei University College of Medicine, \\ Seoul, Republic of Korea
}

\begin{abstract}
Background: Previous studies have demonstrated aberrant Brahma-related gene 1 (BRG1) expression in various tumor types. Increased BRGI expression has recently been shown to correlate with aggressive oncogenic behavior in many different types of human cancer. However, the role of BRG1 in breast cancer development and progression is not fully understood. Materials and Methods: We evaluated BRG1 expression in 224 patients with invasive ductal carcinoma (IDC) of the breast using tissue microarray samples and immunohistochemistry. We also investigated whether BRG1 expression status is associated with clinicopathological characteristics and outcomes of patients with IDC. Results: Among the 224 patients with IDC, 37.5\% (84/224) exhibited high BRG1 expression. IDC exhibited significantly higher BRG1 expression compared to ductal carcinoma in situ $(p=0.009)$ and normal breast tissue $(p=0.005)$. High BRG1 expression in IDC significantly correlated with higher histological grade $(p=0.035)$ and presence of distant metastasis $(p=0.002)$. Furthermore, high BRG1 expression was an independent factor for predicting distant metastasis (relative risk=4.079; $p=0.007$ ). In addition, high BRG1 expression predicted shorter overall $(p=0.011)$ and recurrence-free $(p=0.003)$ survival in patients with IDC. In particular, BRG1 had a significant prognostic value in predicting recurrence-free survival of patients with
\end{abstract}

Correspondence to: Hyun-Soo Kim, Department of Pathology, Severance Hospital, Yonsei University College of Medicine, 50-1, Yonsei-ro, Seodaemun-gu, Seoul 03722, Republic of Korea. Tel: +82 222281794, +82 23620860, e-mail: hyunsookim@yuhs.ac

Key Words: Brahma-related gene 1, breast cancer, invasive ductal carcinoma, distant metastasis, survival.
IDC with lymph node metastasis or stage III disease. Conclusion: BRG1 is involved in the progression and metastasis of breast cancer and can serve as a novel biomarker predictive of distant metastasis and patient outcomes.

Breast cancer is the most common cancer in women, accounting for $23 \%$ of all new cancer cases and $14 \%$ of all cancer-related deaths (1). It is more than twice as common as cancer that occurs in any other part of the body. Invasive ductal carcinoma (IDC) is the most common histological subtype of breast cancer $(2,3)$. Well-established prognostic parameters of IDC, including histological grade, American Joint Committee on Cancer (AJCC) tumor-node-metastasis (TNM) stage, lymphovascular invasion (LVI), estrogen receptor (ER) and progesterone receptor (PR) expression status, and human epithelial growth factor receptor-2 (HER2) gene amplification, provide powerful prognostic implications and help clinicians to decide therapeutic strategies for patients with IDC. In addition, the development of distant metastasis (DM) is one of the most important prognostic factors affecting patient mortality. Although many researchers have investigated potential biomarkers to predict the development of DM in IDC, no reliable biomarker has been identified to date. Therefore, there is an urgent need to establish novel predictive indicators to identify patients who are at higher risk of developing DM. This might enable oncologists to begin tailoring therapeutic strategies to individual patients and to improve their survival.

Many different patterns of aberrant gene expression are involved in tumor development and progression. The mammalian switching/sucrose non-fermenting complexes mediate chromatin remodeling processes that are critical for cellular differentiation and proliferation (4). Brahma-related 
gene-1 (BRG1), the central catalytic subunit of chromatinmodifying enzymatic complexes, disrupts the chromatin architecture of target promoters (5). BRG1 has been implicated in the activation and repression of gene expression through chromatin modulation in various tissues and pathophysiological conditions (5). Previous studies have demonstrated aberrant BRG1 expression in several human tumor types, including glioma, gastric cancer, prostate cancer, and malignant melanoma (6-9). In vitro studies revealed that BRG1 promotes survival and proliferation of melanoma cells by up-regulating the microphthalmiaassociated transcription factor $(10,11)$. Similarly, increased BRG1 expression was shown to be associated with advanced stages of gastric cancer, as well as with tumor progression and invasion in prostate cancer $(7,9)$. Although recent data have suggested that aberrant BRG1 expression is involved in the development and progression of many different types of malignancies, the role of BRG1 in breast cancer remains to be elucidated. In fact, investigations of BRG1 expression in breast cancer tissue specimens are very limited. In this study, we analyzed BRG1 expression patterns in IDC using tissue microarray (TMA) samples and immunohistochemical staining, and we investigated the association between BRG1 and clinicopathological characteristics and outcomes of patients with IDC.

\section{Materials and Methods}

Patients and tissue specimens. We selected 224 cases of IDC and 34 cases of ductal carcinoma in situ (DCIS) from the archival cases in the Kangbuk Samsung Hospital (Seoul, Republic of Korea). All procedures were conducted with a prior approval from the Institutional Review Board of Kangbuk Samsung Hospital (201501-013-001). Formalin-fixed, paraffin-embedded (FFPE) tumor tissue blocks were sectioned, stained with hematoxylin and eosin, and reviewed by two board-certified pathologists. Twelve normal breast tissue samples were used as controls. The following clinicopathological characteristics were included: age of the patients, histologic grade, tumor size, lymph node metastasis (LNM) or DM, stage group, LVI, extensive intraductal component, skin involvement, ER, PR, and HER2 status, triple negativity (ERnegative/PR-negative/HER2-negative), tumor recurrence, follow-up period, and death from IDC. The histological grade was assigned using tubule formation, nuclear pleomorphism, and mitotic counts, based on the modified Bloom-Richardson grading system (12). The stage group at the time of diagnosis was determined based on the AJCC TNM staging system (13). All procedures were conducted with a prior approval from the Institutional Review Board of Kangbuk Samsung Hospital, Seoul, Republic of Korea (2015-01013-001).

Construction of TMA. The surgical specimens were fixed in $10 \%$ buffered formalin, processed, and embedded in paraffin using a standard protocol. All hematoxylin and eosin-stained slides were reviewed, and the two most representative tumor areas were carefully selected and marked on individual FFPE blocks. Two tissue cores ( $2 \mathrm{~mm}$ in diameter) were then obtained from each specimen and manually arrayed in recipient paraffin blocks. The assembly was held in an X-Y position guide with a 1-mm increment between the individual samples, and the instrument was used to create holes in a recipient block with defined array cores. The fit needle was used to transfer the tissue cores into the recipient block. The percentage of tumor volume in each tissue core was greater than $70 \%$. A pair of TMA blocks was made for each individual case.

Immunohistochemistry. Immunohistochemical staining was performed on $3 \mu \mathrm{m}$-thick TMA block sections. Briefly, the sections were dehydrated and deparaffinized in xylene and then rehydrated in a graded series of alcohol solutions. We used primary antibodies against ER (1:200, clone SP1; Lab Vision Corporation, Fremont, CA, USA), PR (1:200, clone PgR 636; DakoCytomation, Glostrup, Denmark), HER2 (1:200, clone SP3, Lab Vision Corporation), and BRG1 (1:100; clone H-88; Santa Cruz Biotechnology, Inc., Santa Cruz, CA, USA). Immunostaining was performed using a compact polymer method (Bond Intense Detection Kit, Leica Biosystems, Newcastle upon Tyne, UK). The primary antibodies were detected with Dako EnVision+ Systems, HRP (DakoCytomation), according to the manufacturer's instructions. The Dako EnVision+ Detection Systems, Peroxidase/DAB (DakoCytomation) was used to perform chromogenic visualization. The slides were then counterstained with hematoxylin and coverslipped. ER and PR status was assessed using the Allred scoring method (14). HER2 expression was evaluated using American Society of Clinical Oncology/College of American Pathologists guideline recommendations (15). In cases with equivocal HER2 staining (score 2), silver in situ hybridization was performed to determine $H E R 2$ gene status. BRG1 staining intensity in the tumor cells was scored on a scale of 0 to 3 : 0 , negative; 1 , weak; 2, moderate; and 3, strong. The percentage of BRG1-positive tumor cells was also classified into one of the four categories: 1 , $0 \%$ to $25 \% ; 2,25 \%$ to $49 \% ; 3,50 \%$ to $74 \%$; and $4,75 \%$ to $100 \%$. The final score was calculated by multiplying the intensity score and the percentage score (16). When a discrepancy occurred between duplicate cores, the higher score was used as the final score. BRG1 immunoreactivity was then classified as low (score 0 to 6) and high (score 8 to 12 ) expression. All slides were examined and scored by two Board-certified pathologists, who were blinded to the clinicopathological data and patient identity. Disagreements between the two pathologists were resolved by consensus.

Statistical analysis. The chi-square test, Fisher's exact test, or linearby-linear association test was performed to determine the correlation between BRG1 expression status and clinicopathological characteristics. Multivariate logistic regression analysis with a backward stepwise elimination method was used to identify the independent predictors of DM. Univariate and multivariate survival analyses were used to examine the prognostic significance of BRG1 expression. Curves for overall (OS) and recurrence-free (RFS) survival were drawn according to the Kaplan-Meier method, and differences were analyzed by applying the log-rank test for univariate survival analysis. Multivariate survival analysis was performed for parameters that achieved statistical significance in univariate survival analysis, using the Cox proportional hazards model [95\% confidence interval (CI)] with a backward stepwise elimination method. Statistical analyses were performed using PASW Statistics for Windows (version 18.0; IBM SPSS Inc., Chicago, IL, USA). Statistical significance was defined as a p-value of less than 0.05 . 


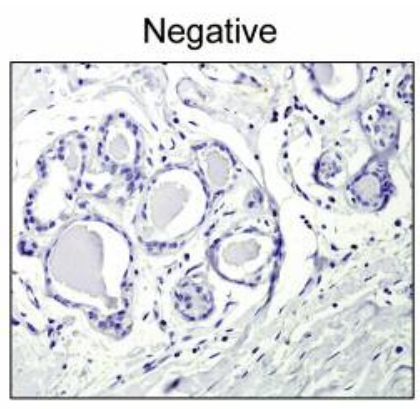

Normal breast
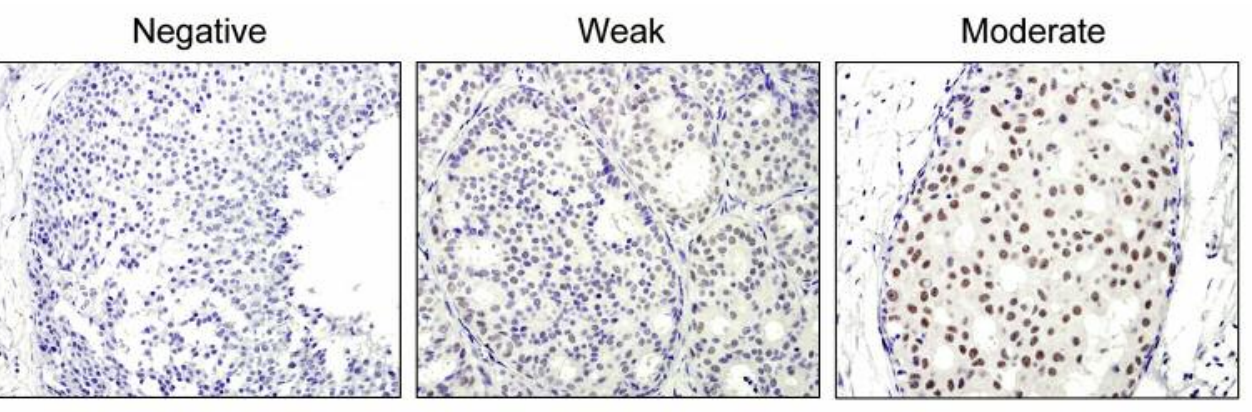

Ductal carcinoma in situ
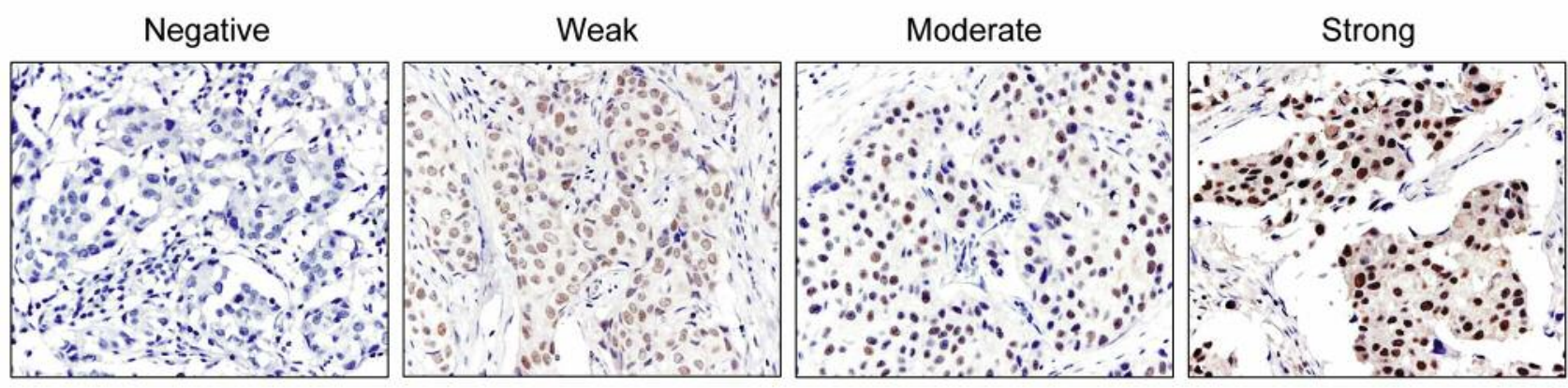

\section{Invasive ductal carcinoma}

Figure 1. Representative photomicrographs of Brahma-related gene 1 (BRG1) immunostaining in normal breast, ductal carcinoma in situ and invasive ductal carcinoma. None of the normal breast tissue samples exhibited BRG1 expression. In both ductal carcinoma in situ and invasive ductal carcinoma, BRG1 was predominantly located in the nuclei of tumor cells. Polymer method. Original magnification, $\times 100$.

\section{Results}

Patient demographics. The median age of patients was 51 years (range $=28-92$ years). Of the 104 patients with LNM, $65(62.5 \%)$ had pN1 disease, $21(20.2 \%)$ had $\mathrm{pN} 2$ disease, and $18(17.3 \%)$ had $\mathrm{pN} 3$ disease. Twenty $(8.9 \%)$ patients had DM in the central nervous system, lung, liver, pancreas, adrenal gland, peritoneum, or bone. Seventy-six patients $(33.9 \%)$ had stage I disease, 106 (47.3\%) had stage II disease, and $42(18.8 \%)$ had stage III disease. LVI was detected in $89(39.7 \%)$ patients. Forty $(17.9 \%)$ patients exhibited extensive intraductal component. Thirty (13.4\%) patients had triple negative tumors. Nine $(4.0 \%)$ patients had died by the time of the last follow-up, with a median time of 48 months from surgery to death. The median follow-up of survivors was 63 months. One hundred and sixteen patients survived more than 5 years. Thirty (13.4\%) patients had tumors that recurred during the follow-up period.

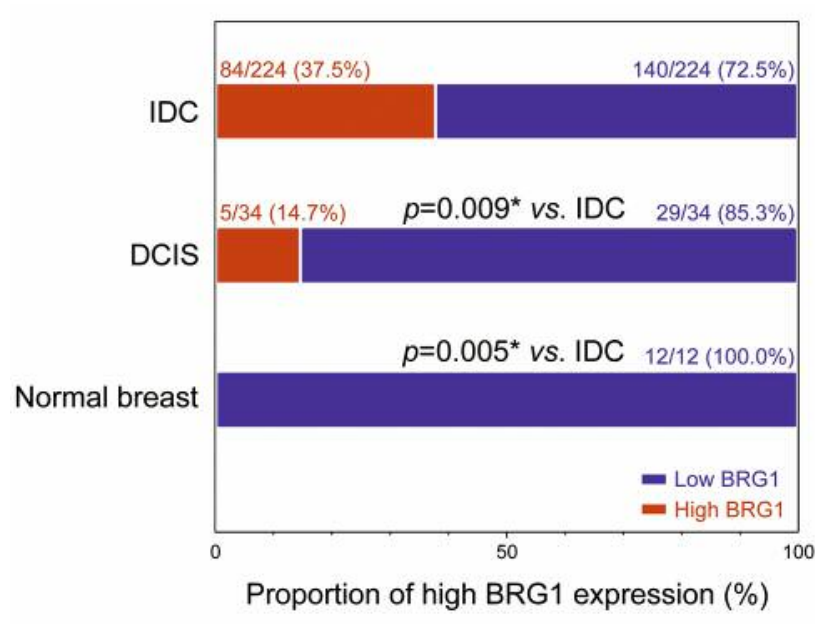

Figure 2. Brahma-related gene 1 (BRG1) expression patterns in normal breast, ductal carcinoma in situ and invasive ductal carcinoma. The frequency of high BRG1 expression was significantly higher in invasive ductal carcinoma than in ductal carcinoma in situ and normal breast tissue. *Significantly different. 
BRG1 expression and its association with clinicopathologic characteristics. Representative photomicrographs of BRG1 immunostaining in normal breast tissue, DCIS and IDC are shown in Figure 1. In 12 normal breast tissue samples, BRG1 immunoreactivity was absent. Among the DCIS tissues, $14.7 \%(5 / 34)$ of the tissues exhibited high BRG1 expression in the nuclei of tumor cells, while $85.3 \%(29 / 34)$ of tissues exhibited low $(48.3 \%$; $14 / 29)$ or no $(51.7 \% ; 15 / 29)$ BRG1 expression. In IDC tissues, BRG1 was observed in the tumor cell nuclei. In a few cases with strong nuclear BRG1 immunoreactivity, faint to weak cytoplasmic staining was noted. Among the 224 IDC tissue samples, 84 (37.5\%) exhibited high BRG1 expression, whereas $140(62.5 \%)$ had low BRG1 expression. Frequency of high BRG1 expression in IDC was significantly higher than that in DCIS $(p=0.009)$ or normal breast tissues $(p=0.005$; Figure 2$)$.

Relationships between BRG1 expression and clinicopathological characteristics of IDC patients are summarized in Table I. Significant associations were observed between high BRG1 expression and higher histological grade $(p=0.035)$ and DM $(p=0.002)$. Although there was a trend toward positive association between BRG1 expression and $\mathrm{T}$ stage, the difference did not quite reach statistical significance $(p=0.065)$.

Factors independently predicting DM. We also examined whether DM is associated with other clinicopathological characteristics and BRG1 expression status (Table II). T Stage $(p=0.017), \mathrm{N}$ stage $(p=0.016)$, PR status $(p=0.037)$, and BRG1 expression $(p=0.002)$ were significantly associated with DM in patients with IDC. To identify the factors that independently predict DM development, these four covariates were entered into multivariate logistic regression analysis. We found that high BRG1 expression was the only independent predictive factor for $\mathrm{DM}(p=0.007$; relative risk $=4.079 ; 95 \% \mathrm{CI}=1.478-11.256$ ).

Factors predicting OS and RFS. Univariate analysis of OS revealed that advanced T stage ( $p=0.026), \operatorname{LNM}(p=0.012)$, DM $(p<0.001)$, advanced stage group $(p<0.001)$, LVI $(p=0.024)$, PR negativity $(p=0.029)$, triple negativity $(p=0.029)$, and high BRG1 expression $(p=0.011)$ significantly predicted poor OS (Table III). The 5-year OS rates were $88.9 \%$ for patients with BRG1-high IDC and 98.4\% for patients with BRG1-low IDC (Figure 3A). Multivariate analysis of OS was performed using T stage, LNM, DM, stage group, LVI, PR status, triple negativity, and BRG1 expression status as covariates. Only DM independently predicted OS $(p<0.001$; Table III). BRG1 expression by itself did not predict OS $(p=0.185)$.

Univariate analysis of RFS revealed that a higher histological grade $(p=0.038)$, advanced T stage $(p=0.017)$, DM $(p<0.001)$, advanced stage group $(p=0.044)$, PR
Table I. Relationship between Brahma-related gene 1 and clinicopathological characteristics.

\begin{tabular}{|c|c|c|c|c|}
\hline \multirow[t]{2}{*}{ Characteristic } & \multirow[t]{2}{*}{ Total } & \multicolumn{2}{|c|}{ BRG1 expression, n (\%) } & \multirow[t]{2}{*}{$p$-Value } \\
\hline & & High $(\%)$ & Low $(\%)$ & \\
\hline \multicolumn{5}{|l|}{ Age (years) } \\
\hline$>51$ & 111 & $38(34.2)$ & $73(65.8)$ & \multirow[t]{2}{*}{0.317} \\
\hline$\leq 51$ & 113 & $46(40.7)$ & $67(59.3)$ & \\
\hline \multicolumn{5}{|l|}{ Histologic grade } \\
\hline 1 & 57 & $12(21.1)$ & 45 (78.9) & \multirow[t]{3}{*}{0.035} \\
\hline 2 & 93 & $42(45.2)$ & $51(54.8)$ & \\
\hline 3 & 74 & $30(40.5)$ & $44(59.5)$ & \\
\hline \multicolumn{5}{|l|}{ Tumor size $(\mathrm{cm})$} \\
\hline$>2$ & 112 & $45(40.2)$ & $67(59.8)$ & \multirow[t]{2}{*}{0.408} \\
\hline$\leq 2$ & 112 & $39(34.8)$ & $73(65.2)$ & \\
\hline \multicolumn{5}{|l|}{ T Stage } \\
\hline pT1 & 112 & $39(34.8)$ & $73(65.2)$ & \multirow[t]{3}{*}{0.065} \\
\hline pT2 & 101 & $36(35.6)$ & $65(64.4)$ & \\
\hline pT3 & 11 & $9(81.8)$ & $2(18.2)$ & \\
\hline \multicolumn{5}{|l|}{ N Stage } \\
\hline pNO & 120 & $43(35.8)$ & $77(64.2)$ & \multirow[t]{4}{*}{0.699} \\
\hline $\mathrm{pN} 1$ & 65 & $24(36.9)$ & $41(63.1)$ & \\
\hline $\mathrm{pN} 2$ & 21 & $12(57.1)$ & $9(42.9)$ & \\
\hline $\mathrm{pN} 3$ & 18 & $5(27.8)$ & $13(72.2)$ & \\
\hline \multicolumn{5}{|l|}{ Distant metastasis } \\
\hline Present & 20 & $14(70.0)$ & $6(30.0)$ & \multirow[t]{2}{*}{0.002} \\
\hline Absent & 204 & $70(34.3)$ & $134(65.7)$ & \\
\hline \multicolumn{5}{|l|}{ TNM stage } \\
\hline I & 76 & $25(32.9)$ & $51(67.1)$ & \multirow[t]{3}{*}{0.133} \\
\hline II & 106 & $39(36.8)$ & $67(63.2)$ & \\
\hline III & 42 & $20(47.6)$ & $22(52.4)$ & \\
\hline \multicolumn{5}{|l|}{ LVI } \\
\hline Present & 89 & $32(36.0)$ & $57(64.0)$ & \multirow[t]{2}{*}{0.698} \\
\hline Absent & 135 & $52(38.5)$ & $83(61.5)$ & \\
\hline \multicolumn{5}{|l|}{ EIC } \\
\hline Present & 40 & $13(32.5)$ & $27(67.5)$ & \multirow[t]{2}{*}{0.471} \\
\hline Absent & 184 & $71(38.6)$ & $113(61.4)$ & \\
\hline Skin involvemen & & & & \\
\hline Present & 5 & $3(60.0)$ & $2(40.0)$ & 0.371 \\
\hline Absent & 197 & $74(37.6)$ & $123(62.4)$ & \\
\hline Not available & 22 & & & \\
\hline ER status & & & & \\
\hline Positive & 161 & $57(35.4)$ & $104(64.6)$ & 0.300 \\
\hline Negative & 63 & $27(42.9)$ & $36(57.1)$ & \\
\hline PR status & & & & \\
\hline Positive & 148 & $50(33.8)$ & $98(66.2)$ & 0.109 \\
\hline Negative & 76 & $34(44.7)$ & $42(55.3)$ & \\
\hline HER2 status & & & & \\
\hline Positive & 59 & $27(45.8)$ & $32(54.2)$ & 0.127 \\
\hline Negative & 165 & $57(34.5)$ & $108(65.5)$ & \\
\hline Triple negative & & & & \\
\hline Yes & 30 & $12(40.0)$ & $18(60.0)$ & 0.761 \\
\hline No & 194 & $72(37.1)$ & $122(62.9)$ & \\
\hline
\end{tabular}

LVI: Lymphovascular invasion; EIC: extensive intraductal component; ER: estrogen receptor; PR: progesterone receptor; HER2: human epidermal growth factor receptor 2 . 
Table II. Factors predicting distant metastasis in patients with invasive ductal carcinoma of the breast.

\begin{tabular}{|c|c|c|c|c|c|}
\hline \multirow[t]{3}{*}{ Characteristic } & \multirow{2}{*}{\multicolumn{2}{|c|}{ Distant metastasis, $\mathrm{n}(\%)$}} & \multirow{3}{*}{$\begin{array}{c}\text { Univariate } \\
p \text {-Value }\end{array}$} & \multicolumn{2}{|c|}{ Multivariate } \\
\hline & & & & \multirow[t]{2}{*}{ RR (95\% CI) } & \multirow[t]{2}{*}{$p$-Value } \\
\hline & Present & Absent & & & \\
\hline \multicolumn{6}{|l|}{ Age, years } \\
\hline$>51$ & $6(5.4)$ & $105(94.6)$ & 0.067 & NA & \\
\hline$\leq 51$ & $14(12.4)$ & 99 (87.6) & & & \\
\hline \multicolumn{6}{|c|}{ Histological grade } \\
\hline 1 & $2(3.5)$ & $55(96.5)$ & 0.092 & NA & \\
\hline 2 & $9(9.7)$ & $84(90.3)$ & & & \\
\hline 3 & $9(12.2)$ & $65(87.8)$ & & & \\
\hline \multicolumn{6}{|c|}{ Tumor size $(\mathrm{cm})$} \\
\hline$>2$ & $12(10.7)$ & $100(89.3)$ & 0.349 & NA & \\
\hline$\leq 2$ & $8(7.1)$ & $104(92.9)$ & & & \\
\hline \multicolumn{6}{|l|}{ T Stage } \\
\hline pT1 & $8(7.1)$ & $104(92.9)$ & 0.017 & 1.049 & 0.927 \\
\hline pT2 & $7(6.9)$ & $94(93.1)$ & & $(0.382-2.881)$ & \\
\hline pT3 & $5(45.5)$ & $6(54.5)$ & & & \\
\hline \multicolumn{6}{|l|}{ N Stage } \\
\hline pNO & $7(5.8)$ & $113(94.2)$ & 0.016 & 2.386 & 0.086 \\
\hline $\mathrm{pN} 1$ & $5(7.7)$ & $60(92.3)$ & & $(0.885-6.436)$ & \\
\hline $\mathrm{pN} 2$ & $5(23.8)$ & $16(76.2)$ & & & \\
\hline $\mathrm{pN} 3$ & $3(16.7)$ & $15(83.3)$ & & & \\
\hline \multicolumn{6}{|l|}{ LVI } \\
\hline Present & $12(13.5)$ & $77(86.5)$ & 0.052 & NA & \\
\hline Absent & $8(5.9)$ & $127(94.1)$ & & & \\
\hline \multicolumn{6}{|l|}{ EIC } \\
\hline Present & $1(2.5)$ & $39(97.5)$ & 0.138 & NA & \\
\hline Absent & $19(10.3)$ & $165(89.7)$ & & & \\
\hline \multicolumn{6}{|l|}{ ER status } \\
\hline Positive & $13(8.1)$ & $148(91.9)$ & 0.474 & NA & \\
\hline Negative & $7(11.1)$ & $56(88.9)$ & & & \\
\hline \multicolumn{6}{|l|}{ PR status } \\
\hline Positive & $9(6.1)$ & $139(93.9)$ & 0.037 & 2.441 & 0.070 \\
\hline Negative & $11(14.5)$ & $65(85.5)$ & & $(0.931-6.398)$ & \\
\hline \multicolumn{6}{|l|}{ HER2 status } \\
\hline Positive & $4(6.8)$ & $55(93.2)$ & 0.603 & NA & \\
\hline Negative & $16(9.7)$ & $149(90.3)$ & & & \\
\hline \multicolumn{6}{|l|}{ Triple negativity } \\
\hline Yes & $5(16.7)$ & $25(83.3)$ & 0.110 & NA & \\
\hline No & $15(7.7)$ & $179(92.3)$ & & & \\
\hline \multicolumn{6}{|c|}{ BRG1 expression } \\
\hline High & $14(16.7)$ & $70(83.3)$ & 0.002 & 4.079 & 0.007 \\
\hline Low & $6(4.3)$ & $134(95.7)$ & & $(1.478-11.256)$ & \\
\hline
\end{tabular}

LVI: Lymphovascular invasion; EIC: extensive intraductal component; ER: estrogen receptor; PR: progesterone receptor; HER2: human epidermal growth factor receptor 2; RR: relative risk; CI: confidence interval; NA: not applicable; BRG1: Brahma-related gene 1.

negativity $(p=0.019)$, triple negativity $(p=0.003)$, and high BRG1 expression $(p=0.003)$ were significant predictors of poor RFS (Table III). The 3- and 5-year RFS rates were $83.0 \%$ and $77.5 \%$, respectively, for patients with BRG1-high IDC and $95.4 \%$ and $91.0 \%$, respectively, for patients with BRG1-low IDC, respectively (Figure 3B). In multivariate analysis, advanced $\mathrm{T}$ stage $(p=0.043)$, presence of $\mathrm{DM}$ $(p<0.001)$, and triple negativity $(p=0.025)$ were independent predictors of shorter RFS (Table III). The hazard ratio of high BRG1 expression for RFS (2.236; 95\% confidence interval $=0.908-4.566)$ was similar to that for $\mathrm{T}$ stage $(2.385$; 95\% $\mathrm{CI}=1.027-5.540)$ and triple negativity $(2.572 ; 95 \%$ $\mathrm{CI}=1.126-5.875)$, but BRG1 expression status was not a significant independent predictor of RFS $(p=0.084)$. 

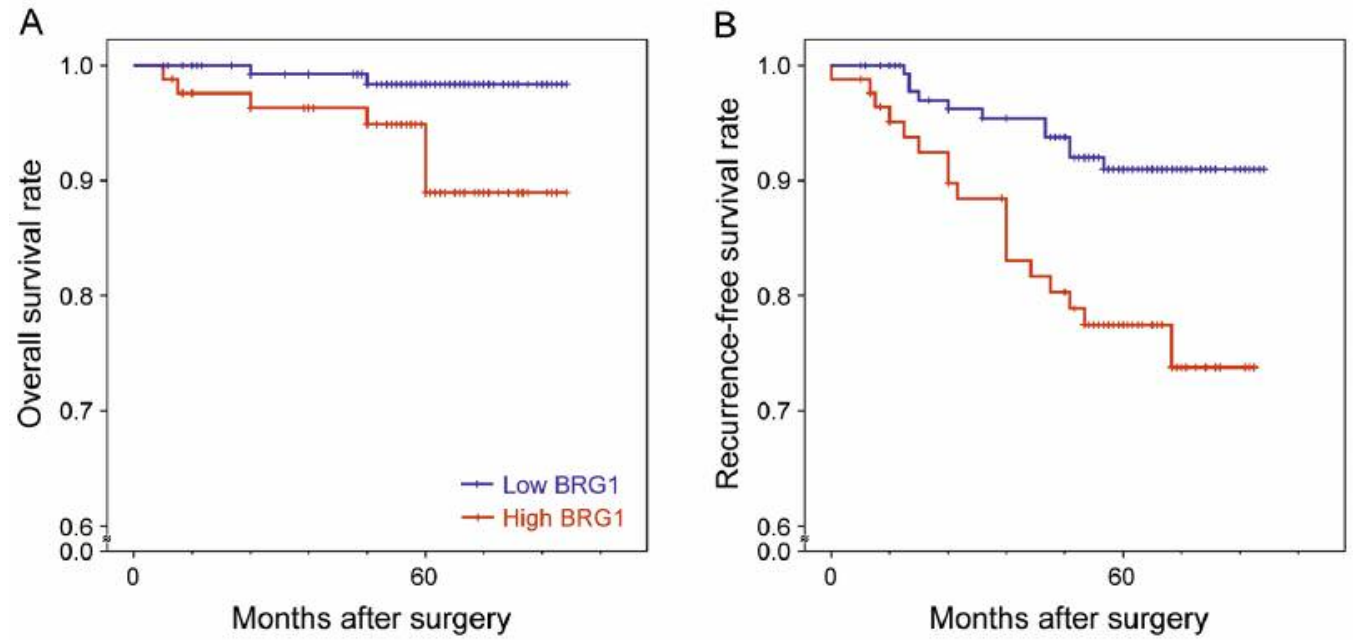

Figure 3. Prognostic significance of Brahma-related gene 1 (BRG1) expression in patients with invasive ductal carcinoma. Kaplan-Meier curves illustrating overall survival (A) and recurrence-free survival among patients with invasive ductal carcinoma with respect to BRG1 expression status. Patients with high BRG1 expression had shorter overall survival $(p=0.011)$ and recurrence-free survival $(p=0.003)$ compared to those with low expression.

Table III. Factors predicting shortened overall and recurrence-free survival of patients with invasive ductal carcinoma of the breast Characteristic

\begin{tabular}{|c|c|c|c|c|c|c|}
\hline & \multicolumn{3}{|c|}{ Overall survival } & \multicolumn{3}{|c|}{ Recurrence-free survival } \\
\hline & \multirow{2}{*}{$\begin{array}{c}\text { Univariate } \\
p \text {-Value }\end{array}$} & \multicolumn{2}{|c|}{ Multivariate } & \multirow{2}{*}{$\frac{\text { Univariate }}{p \text {-Value }}$} & \multicolumn{2}{|c|}{ Multivariate } \\
\hline & & $\operatorname{HR}(95 \% \mathrm{CI})$ & $p$-Value & & $\operatorname{HR}(95 \% \mathrm{CI})$ & $p$-Value \\
\hline Age (years): $>51 v s . \leq 51$ & 0.747 & NA & & 0.338 & NA & \\
\hline Histological grade: $3-2$ vs. 1 & 0.069 & NA & & 0.038 & $1.352(0.371-4.927)$ & 0.647 \\
\hline T Stage: pT2-3 vs. pT1 & 0.026 & $4.329(0.536-34.999)$ & 0.169 & 0.017 & $2.385(1.027-5.540)$ & 0.043 \\
\hline Lymph node metastasis: Present $v s$. absent & 0.012 & $6.157(0.752-50.411)$ & 0.090 & 0.173 & NA & \\
\hline Distant metastasis: Present $v s$. absent & $<0.001$ & $11.909(3.145-45.101)$ & $<0.001$ & $<0.001$ & $11.509(5.275-25.112)$ & $<0.001$ \\
\hline TNM stage: III $v s$. I-II & $<0.001$ & $1.092(0.164-7.267)$ & 0.927 & 0.044 & $1.071(0.458-2.503)$ & 0.875 \\
\hline LVI: Present $v s$. absent & 0.024 & $1.900(0.348-10.356)$ & 0.458 & 0.174 & NA & \\
\hline ER status: Negative $v s$. positive & 0.213 & NA & & 0.132 & NA & \\
\hline PR status: Negative $v s$. positive & 0.029 & $1.039(0.160-6.738)$ & 0.968 & 0.019 & $0.775(0.287-2.091)$ & 0.615 \\
\hline HER2 status: Negative $v s$. positive & 0.248 & NA & & 0.353 & NA & \\
\hline Triple negativity: Yes $v s$. no & 0.029 & $3.409(0.804-14.455)$ & 0.096 & 0.003 & $2.572(1.126-5.875)$ & 0.025 \\
\hline BRG1 expression: High $v s$. low & 0.011 & $3.122(0.580-16.807)$ & 0.185 & 0.003 & $2.236(0.908-4.566)$ & 0.084 \\
\hline
\end{tabular}

LVI: Lymphovascular invasion; ER: estrogen receptor; PR: progesterone receptor; HER2: human epidermal growth factor receptor 2; BRG1: Brahmarelated gene 1; HR: hazard ratio; CI: confidence interval; NA: not applicable.

Moreover, we evaluated the prognostic value of BRG1 expression in selected patient subgroups. Firstly, we divided the patients into two groups according to LN status (nodenegative and node-positive). In the node-negative group, we did not find any difference in OS ( $p=0.455$; Figure $4 \mathrm{~A})$ or RFS ( $p=0.297$; Figure 4C) with respect to BRG1 expression. In contrast, in the node-positive group, there were significant differences in both OS ( $p=0.004$; Figure 4B) and RFS ( $p=0.002$; Figure 4D) between patients with BRG1-high IDC and BRG1-low IDC. Secondly, when the patients were classified into three groups according to stage (stage I, II, and III), the difference in RFS of patients with BRG1-high IDC and BRG1-low IDC was statistically significant in the stage III group ( $p=0.005$; Figure $5 \mathrm{C}$ ). The 5 -year RFS of 
A

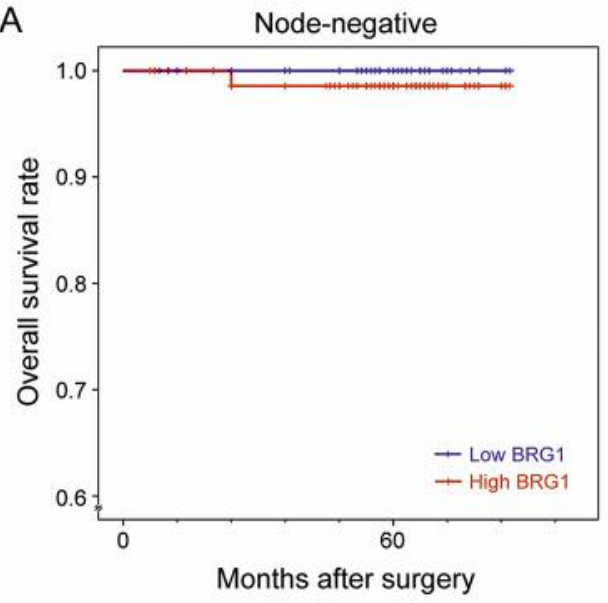

C

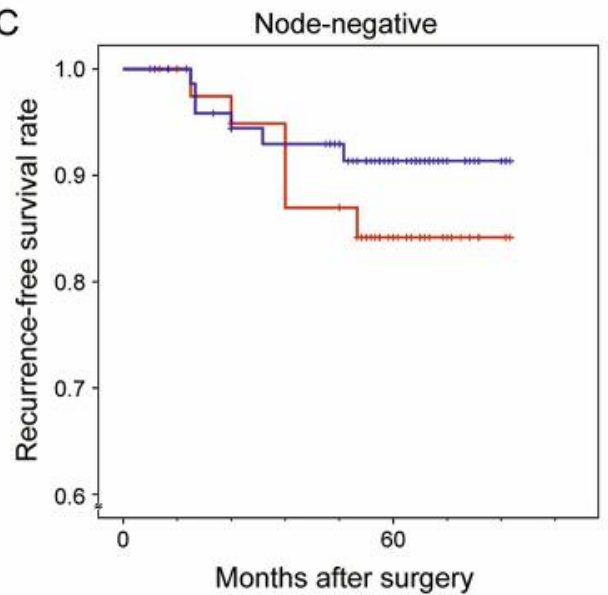

B

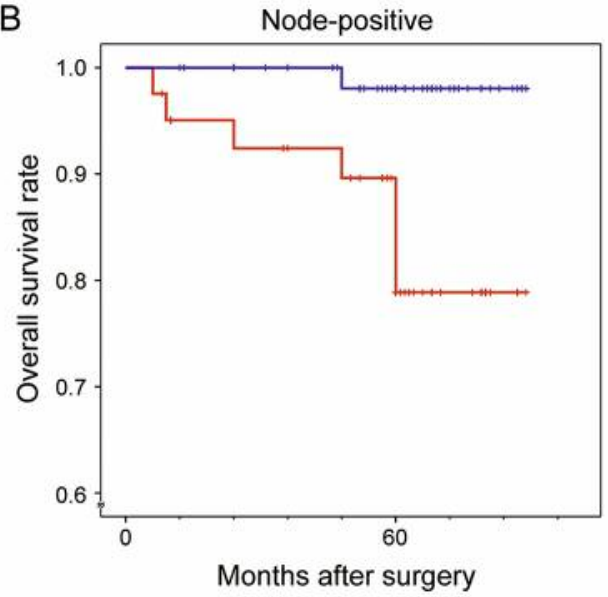

D

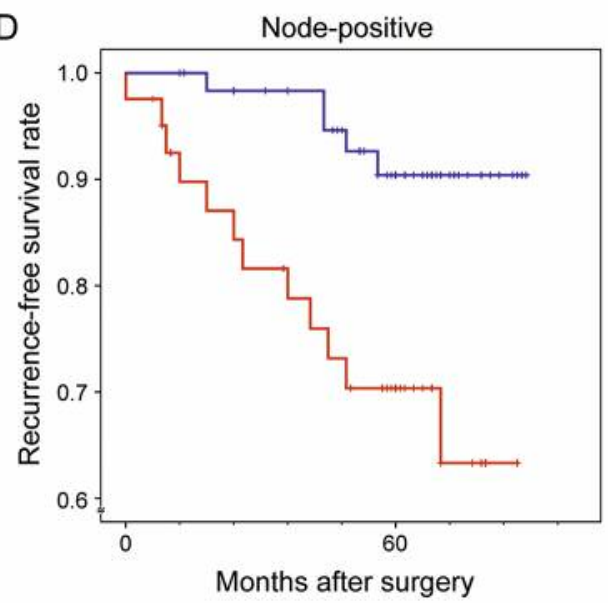

Figure 4. Prognostic significance of Brahma-related gene 1 (BRG1) expression in specific patient subgroups based on lymph node metastasis status. Kaplan-Meier curves showing overall survival $(A, B)$ and recurrence-free survival $(C, D)$ of patients with node-negative (A, $C)$ and node-positive $(B, D)$ invasive ductal carcinoma. In patients with lymph node metastasis, high BRG1 expression was associated with significantly shorter overall survival $(p=0.004)$ and recurrence-free survival $(p=0.002)$. In patients without lymph node metastasis, the differences in overall and recurrencefree survival with regard to BRG1 expression status were not statistically significant $(p=0.455$ and $p=0.297)$.

A

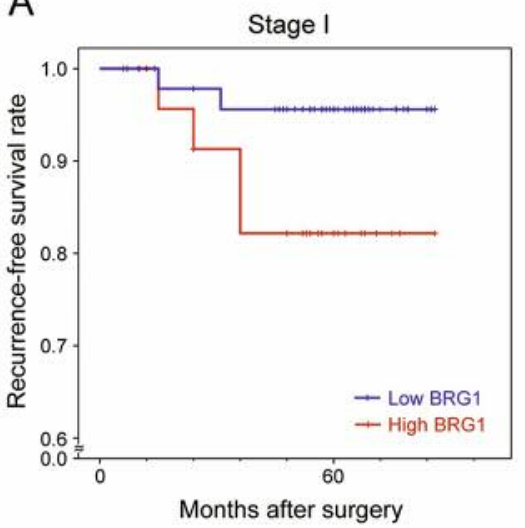

B

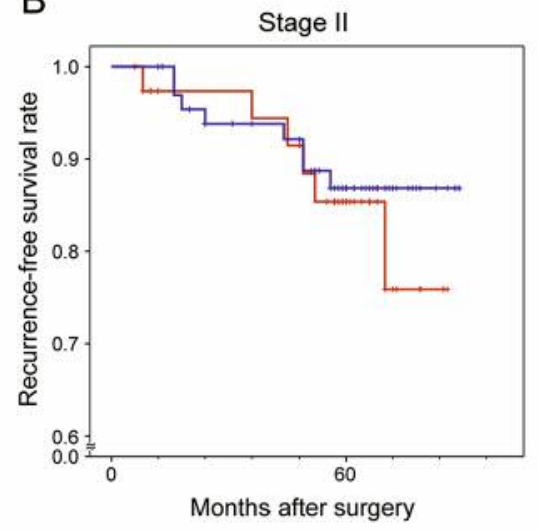

C



Figure 5. Prognostic significance of Brahma-related gene 1 (BRG1) expression in specific subgroups based on the stage group. Kaplan-Meier curves illustrating recurrence-free survival of patients with stage I $(A)$, stage II $(B)$ and stage III $(C)$ invasive ductal carcinoma. In the stage I and II subgroups, patients with high expression of BRG1 exhibited shorter recurrence-free survival than those with low expression but the difference between the two subgroups did not reach statistical significance ( $p=0.071$ and $p=0.551$, respectively). In contrast, in the stage III subgroup, the recurrencefree survival of patients with stage III IDC with high expression of BRG1 was significantly shorter than that of those with low expression ( $p=0.005$ ). 
patients with BRG1-high stage I IDC was shorter than that of those with BRG1-low stage I IDC, but the difference did not reach statistical significance ( $p=0.071$; Figure 5A). In patients with stage II IDC, the difference in RFS with respect to BRG1 expression status was not significant $(p=0.551$; Figure 5B).

\section{Discussion}

Although there has been considerable interest in the role of BRG1 in tumorigenesis, studies have provided contradictory results, with both pro-oncogenic and tumor-suppressive roles being reported. In fact, there are major discrepancies between early and recent investigations. Early studies suggest that the $B R G 1$ gene is frequently silenced in several cancer cell lines and that BRG1 functions as a tumor suppressor. BRG1 was found to be frequently lost in intraductal papillary mucinous neoplasm of the pancreas and it suppressed the formation of both intraductal papillary mucinous neoplasm and pancreatic cancer (17). BRGl expression was also lost in $15-20 \%$ of non-small cell lung carcinomas (18). Consistent with these data, cancer cell lines from the lung, adrenal gland, uterine cervix, and pancreas showed loss of $B R G l$ expression (18, 19). However, recent investigations suggest otherwise. Knockdown of $B R G 1$ suppressed cell proliferation in human melanoma and colorectal cancer cell lines $(8,20)$. BRG1 was found to interact with the microphthalmia-associated transcription factor, an oncoprotein, to promote melanoma survival and proliferation $(10,11)$. Silencing $B R G 1$ in glioma cell lines inhibited cell growth, migration, and invasion (6). In addition, a recent in vitro study using a pancreatic cancer cell line demonstrated that BRG1 promoted both prooncogenic and tumor-suppressive activities at distinct stages of pancreatic carcinogenesis (21). In pancreatic ductal epithelium, loss of $B R G 1$ promoted dedifferentiation of ductal epithelium that precedes neoplastic transformation, whereas at an advanced stage, re-expression of BRG1 enhanced tumorigenesis and epithelial-mesenchymal transition, suggesting stage-specific roles of $B R G 1$ (21). There are also conflicting reports on the correlation between BRG1 expression and patient survival. In patients with non-small cell lung carcinomas, loss of $B R G 1$ expression correlated with shortened survival $(18,22)$. Some studies have shown that low BRG1 expression correlates with poor prognosis in patients with pancreatic cancer $(17,21,23)$. However, a recent study demonstrated that high BRG1 expression is associated with shortened survival of patients with pancreatic cancer (24). Therefore, it is difficult to assess whether BRG1 is a tumor suppressor or an oncogene. The possibility that the biological significance of BRG1 differs during tumor development according to cell or tissue type cannot be excluded. Further investigations are warranted to clarify the molecular mechanism of BRG1 in carcinogenesis.
Currently, limited information is available in the literature regarding the expression and regulation of BRG1 in breast cancer. To our knowledge, only one study has examined BRG1 expression in breast cancer tissue samples (25). Although a previous study reported the frequency and prognostic implications of BRG1 expression in patients with IDC, there are no data available regarding BRG1 expression in nonneoplastic breast tissues and DCIS lesions. In this study, we found that BRG1 expression was significantly increased in IDC tissues compared with DCIS and normal breast tissues. Our result is consistent with the previous findings of several studies, which demonstrate that malignant tumor tissues exhibit significantly increased BRG expression compared with nonneoplastic, benign, or preinvasive lesions. In primary and metastatic melanoma tissues, BRG1 immunoreactivity was increased compared with dysplastic nevi (8). BRG1 expression levels in colorectal carcinoma tissues were significantly higher than those in adenomas or normal colonic mucosa (20). Moreover, compared with normal brain tissues, glioma tissues exhibited significantly increased BRG1 levels (6). Taken together, these data suggest that BRG1 expression is a prooncogenic factor in tumor development.

We also examined the association between BRG1 expression and the clinicopathological characteristics of patients with IDC. There was a significant relationship between high BRG1 expression and higher histological grade and DM. These findings suggest that BRG1 expression is involved in tumor progression and metastasis of IDC. This is in agreement with previous data demonstrating that increased BRG1 expression is associated with aggressive oncogenic behavior, such as advanced stage and invasiveness of gastric and prostate cancer $(7,9)$. Stage at the time of initial diagnosis is a strong predictor of DM in patients with IDC. Women whose tumors are locally advanced or spread regionally beyond the breast are much more likely to develop metastases than those with localized tumors. However, as many as $30 \%$ of the patients with localized IDC experience tumor recurrence, and many of these patients will develop DM. In this regard, there is a need for developing criteria based on biological determinants for better and early stratificiation of patients according to the risk of metastatic recurrence. Consequently, this would also help identify patients who should be monitored frequently and may benefit from additional treatment strategies. In this study, high BRG1 expression was the only independent predictor of DM. The extent of LNM is the most important prognostic factor in breast cancer and one of the main determinants for predicting tumor progression and aggressive oncogenic behavior (26). Surprisingly in this study, the relative risk of DM associated with high BRG1 expression (4.079) was higher than the risk associated with $\mathrm{N}$ stage (2.386). To the best of our knowledge, the use of BRG1 expression to predict DM has not been previously investigated. Our 
finding suggests that BRG1 immunostaining provides clinically useful information for patients with IDCs, and BRG1 expression is a strong and novel predictive marker for the identification of patients at high risk of developing DM.

We further observed that patients with IDC whose tumors exhibited high BRG1 expression had shorter OS and RFS than those with BRG1-low IDCs. These results are in agreement with a previous study demonstrating that high BRG1 expression is associated with both decreased overall and disease-specific 5-year survival of patients with IDC (25). These results also suggest that BRG1 expression in IDC can be used as a novel prognostic marker for poor outcomes in patients with IDC. Although the TNM staging system successfully grades patients with respect to their prognosis according to the clinicopathological characteristics, it does not provide critical information that may influence treatment strategy. Many biomarkers have been investigated to overcome the limitations of the traditional system and have shown potential predictive significance. However, to date, reliable biomarkers that can stratify patients with IDC with LNM are substantially limited. In our analysis, high BRG1 expression had a clear prognostic value for both OS and RFS in patients with node-positive IDC. Similarly, BRG1 had significant prognostic implications for RFS of patients with stage III IDC. These data imply that BRG1 expression might be a predictive tool for identifying patients with LNM or stage III disease who are at high risk of recurrence.

In conclusion, BRG1 expression in IDC tissue was significantly higher than that in DCIS and normal breast tissue. High BRG1 expression predicted DM and shortened survival. Changes in BRG1 regulation may potentially promote tumor development, progression, and metastasis of IDC, and the level of BRG1 expression may serve as a predictive biomarker for DM and patient outcomes.

\section{References}

1 Jemal A, Bray F, Center MM, Ferlay J, Ward E and Forman D: Global cancer statistics. CA Cancer J Clin 61: 69-90, 2011.

2 Li CI, Uribe DJ and Daling JR: Clinical characteristics of different histologic types of breast cancer. Br J Cancer 93: 10461052, 2005.

3 Li CI, Anderson BO, Daling JR and Moe RE: Trends in incidence rates of invasive lobular and ductal breast carcinoma. JAMA 289: 1421-1424, 2003

4 Reisman D, Glaros S and Thompson EA: The SWI/SNF complex and cancer. Oncogene 28: 1653-1668, 2009.

5 Trotter KW and Archer TK: The BRG1 transcriptional coregulator. Nucl Recept Signal 6: e004, 2008.

6 Bai J, Mei PJ, Liu H, Li C, Li W, Wu YP, Yu ZQ and Zheng JN: BRG1 expression is increased in human glioma and controls glioma cell proliferation, migration and invasion in vitro. $\mathbf{J}$ Cancer Res Clin Oncol 138: 991-998, 2012.

7 Sun A, Tawfik O, Gayed B, Thrasher JB, Hoestje S, Li C and Li B: Aberrant expression of SWI/SNF catalytic subunits
BRG1/BRM is associated with tumor development and increased invasiveness in prostate cancers. Prostate 67: 203-213, 2007.

8 Lin $\mathrm{H}$, Wong RP, Martinka M and Li G: BRG1 expression is increased in human cutaneous melanoma. Br J Dermatol 163: 502-510, 2010

9 Sentani K, Oue N, Kondo H, Kuraoka K, Motoshita J, Ito R, Yokozaki $\mathrm{H}$ and Yasui $\mathrm{W}$ : Increased expression but not genetic alteration of BRG1, a component of the SWI/SNF complex, is associated with the advanced stage of human gastric carcinomas. Pathobiology 69: 315-320, 2001

10 de la Serna IL, Ohkawa Y, Higashi C, Dutta C, Osias J, Kommajosyula $\mathrm{N}$, Tachibana $\mathrm{T}$ and Imbalzano $\mathrm{AN}$ : The microphthalmia-associated transcription factor requires SWI/SNF enzymes to activate melanocyte-specific genes. J Biol Chem 281: 20233-20241, 2006.

11 Keenen B, Qi H, Saladi SV, Yeung M and de la Serna IL: Heterogeneous SWI/SNF chromatin remodeling complexes promote expression of microphthalmia-associated transcription factor target genes in melanoma. Oncogene 29: 81-92, 2010.

12 Lakhani SR, Ellis IO, Schnitt SJ, Tan PH and van de Vijver MJ: WHO Classification of Tumours of the Breast. Lyon, France: IARC, 2012.

13 Edge SB, Byrd DR, Compton CC, Fritz AG, Greene FL and Trotti A: AJCC Cancer Staging Manual. New York, NY, USA: Springer, 2010.

14 Harvey JM, Clark GM, Osborne CK and Allred DC: Estrogen receptor status by immunohistochemistry is superior to the ligand-binding assay for predicting response to adjuvant endocrine therapy in breast cancer. J Clin Oncol 17: 1474-1481, 1999.

15 Wolff AC, Hammond ME, Hicks DG, Dowsett M, McShane LM, Allison KH, Allred DC, Bartlett JM, Bilous M, Fitzgibbons P, Hanna W, Jenkins RB, Mangu PB, Paik S, Perez EA, Press MF, Spears PA, Vance GH, Viale $G$ and Hayes DF: Recommendations for human epidermal growth factor receptor 2 testing in breast cancer: American Society of Clinical Oncology/College of American Pathologists clinical practice guideline update. Arch Pathol Lab Med 138: 241-256, 2014.

16 Remmele W and Stegner HE: Recommendation for uniform definition of an immunoreactive score (IRS) for immunohistochemical estrogen receptor detection (ER-ICA) in breast cancer tissue. Pathologe 8: 138-140, 1987.

17 von Figura G, Fukuda A, Roy N, Liku ME, Morris Iv JP, Kim GE, Russ HA, Firpo MA, Mulvihill SJ, Dawson DW, Ferrer J, Mueller WF, Busch A, Hertel KJ and Hebrok M: The chromatin regulator Brg1 suppresses formation of intraductal papillary mucinous neoplasm and pancreatic ductal adenocarcinoma. Nat Cell Biol 16: 255-267, 2014.

18 Reisman DN, Sciarrotta J, Wang W, Funkhouser WK and Weissman BE: Loss of BRG1/BRM in human lung cancer cell lines and primary lung cancers: correlation with poor prognosis. Cancer Res 63: 560-566, 2003.

19 Decristofaro MF, Betz BL, Rorie CJ, Reisman DN, Wang W and Weissman BE: Characterization of SWI/SNF protein expression in human breast cancer cell lines and other malignancies. J Cell Physiol 186: 136-145, 2001.

20 Watanabe T, Semba S and Yokozaki H: Regulation of PTEN expression by the SWI/SNF chromatin-remodelling protein BRG1 in human colorectal carcinoma cells. Br J Cancer 104: 146-154, 2011 
21 Roy N, Malik S, Villanueva KE, Urano A, Lu X, Von Figura G, Seeley ES, Dawson DW, Collisson EA and Hebrok M: Brg1 promotes both tumor-suppressive and oncogenic activities at distinct stages of pancreatic cancer formation. Genes Dev 29: 658-671, 2015.

22 Fukuoka J, Fujii T, Shih JH, Dracheva T, Meerzaman D, Player A, Hong K, Settnek S, Gupta A, Buetow K, Hewitt S, Travis WD and Jen J: Chromatin remodeling factors and BRM/BRG1 expression as prognostic indicators in non-small cell lung cancer. Clin Cancer Res 10: 4314-4324, 2004.

23 Dal Molin M, Hong SM, Hebbar S, Sharma R, Scrimieri F, de Wilde RF, Mayo SC, Goggins M, Wolfgang CL, Schulick RD, Lin MT, Eshleman JR, Hruban RH, Maitra A and Matthaei H: Loss of expression of the SWI/SNF chromatin remodeling subunit BRG1/SMARCA4 is frequently observed in intraductal papillary mucinous neoplasms of the pancreas. Hum Pathol 43: 585-591, 2011.
24 Liu X, Tian X, Wang F, Ma Y, Kornmann M and Yang Y: BRG1 promotes chemoresistance of pancreatic cancer cells through crosstalking with Akt signalling. Eur J Cancer 50: 2251-2262, 2014.

25 Bai J, Mei P, Zhang C, Chen F, Li C, Pan Z, Liu H and Zheng $\mathrm{J}$ : BRG1 is a prognostic marker and potential therapeutic target in human breast cancer. PLoS One 8: e59772, 2013.

26 Rack B, Janni W, Gerber B, Strobl B, Schindlbeck C, Klanner E, Rammel G, Sommer H, Dimpfl T and Friese K: Patients with recurrent breast cancer: does the primary axillary lymph node status predict more aggressive tumor progression? Breast Cancer Res Treat 82: 83-92, 2003.

Received July 20, 2016

Revised August 2, 2016

Accepted August 4, 2016 